include programme management and outreach activity costs. Incremental cost-effectiveness analysis allows us to identify the most cost-effective NGO/CBO model for STI service delivery.

Results Most of the NGO/CBOs served more than 2,000 HRIs. However, when standardised to reflect a population of 1,000 HRIs, the programme operated clinic with outreach model was able to deliver STI control services at a lower cost per STI consultation, than alternative models. Incremental cost-effectiveness analysis of alternative STI service models with regards to coverage of STI screening \& syphilis testing, show this model to be the most costeffective model.

Conclusions For larger NGO/CBOs, use of outreach is critical to obtain operational efficiencies. Program operated clinics with outreach were found to be the most cost-effective model, probably due to increased access to scattered high-risk populations through effective outreach activities and continuity of care.

\section{P6.054 CONDOM AND STI A GAZE WORD - TO PREVENT STIS}

doi:10.1136/sextrans-2013-051184.1207

S M Alam. Save the Children, Dhaka, Bangladesh

Background Bangladesh is culturally and religiously conservative. Condom use rate among the married couple is poor. Young people's access to condom is restricted. The perception about condom use in Bangladeshi society is a barrier device to "sexual pleasure". This pervasive claim of "reduced pleasure" is a diverse and complex phenomenon explored through men's statements. Bangladesh has a low HIV prevalence rate with less than $0.1 \%$ in the general population, as there are no changes of HIV prevalence the surrogate markers of risk for active syphilis (unsafe sex) and antibodies of HCV - are of relevance. Among female sex workers HIV prevalence in different setting is $<1 \%$ except in one border town area.

Bangladesh had received 3 grants on HIV/AIDS from the Global Fund to fight AIDS, Tuberculosis and Malaria in Round 2 from 2004-2009, Round 6 from 2007-2012 and Rolling Continuation Channel (RCC) from 2009-2015 on prevention, care, and treatment for the MARP's.

Methods Three surveys conducted Project baseline survey 2005, end line survey 2008 of Prevention of HIV/AIDS among young people in Bangladesh and Mid-Term Survey 2012 on RCC Program Expanding HIV/AIDS Prevention in Bangladesh funded by the Global Fund.

Results Over the period \% of young women and men aged 15-24 yrs who correctly identify at least two ways of preventing HIV transmission knowledge increased over the period 22.6\% (2005); $43.8 \%$ (2008) and $84.8 \%$ (2012) however, \% of female sex workers reporting the use of a condom with their most recent client (Street, Residence \& Hotel) 66.7\% (2006-2007) which is increased 95.5\% (2012) in the mid-term survey of RCC programme.

Conclusion The strategic intervention of GFATM funded HIV programme in Bangladesh achieved the remarkable success.

\section{P6.055 MONITORING HIV RESISTANCE: FIRST BRAZILIAN EXPERIENCE WITH CLINICAL INDICATORS IN A PUBLIC CARE UNIT AT AMAZON STATE}

doi:10.1136/sextrans-2013-051184.1208

'R S M Oliveira, ' $\mathrm{J}$ Gonçalves, ${ }^{2} \mathrm{~K}$ Abtibol. ${ }^{1}$ Tropical Medicine Foundation Heitor Vieira

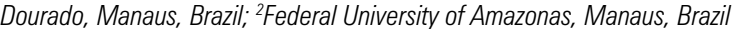

Background World Health Organization has provided countries with "Early Warning Indicators" to monitor ART sites and minimise the emergence of drug resistance, using routine information from medical and pharmacy records. In Brazil the percentage of multiexperienced people is about $8 \%$, at brazilian universal free access, which concerns about complications in quality of life related to therapeutic failure. We evaluated two cohorts at a public health care site at Amazonas state,to propose the indicators validation on this population.

Methods Through electronic local system and following the WHO methodology, patients who started ART treatment twelve months ago, and received care at the unit, were selected for WHO sample size indicated. The research resulted in 315 adults.

Results As results were compared to the original WHO indicators for EWI 1 (percentage of ART prescribing practises with appropriate first-line regimen) at the studied site were $91 \%$ and $95 \%$, for 2009 and 2010 respectively, WHO recommends $100 \%$ for this indicator. EWI 2 (patients lost of follow-up 12 months after ART initiation): 30\% (2009) and 36\% (2010) (WHO = less than $20 \%$ ). EWI 3 (patients on appropriate first-line regimen 12 months after ART initiation): 40\% (2009) and 49\% (2010); (WHO = up to $70 \%$ ). About $75 \%$ picked up ART drugs on time (EWI 4), instead WHO recommends up to $90 \%$.EWI 8 (patients with viral load supression after 12 months of ART): $31 \%$ for 2009 , and $30 \%$ (2010),WHO recommends up to $70 \%$

Conclusions EWI were implemented in other Latin America countries to determine a valid tool compared to viral load. Brazil does not have yet a national monitoring system to monitor sites. This one, located at an HIV late diagnosis area showed deficit in maintenance in care. The managers need to improve monitoring adherence and prevent HIV resistance by therapeutic failure, since there was not any local indicator which achieved WHO recommendations.

\section{P6.056 KNOWLEDGE AND ATTITUDE IN RELATION TO HIV/AIDS AMONG NURSES WORKING AT MATERNITY WARD, BPKIHS, NEPAL}

doi:10.1136/sextrans-2013-051184.1209

S Lama, K Vijayalakshmi, N Pokharel. BP Koirala Institute of Health Sciences, Dharan, Nepal

Background People with HIV frequently encountered discrimination while seeking and receiving health care services. The knowledge and attitude of health care professionals influence the willingness and ability of people with HIV to access care and the quality of care they receive.

Aims To assess the HIV/Aids related knowledge and attitude among nurses working at maternity ward of BPKIHS.

Methods and Materials: A descriptive cross sectional study was conducted in maternity ward of B.P. Koirala Institute of Health Sciences. Purposive sampling technique was used for selection of samples. Data was collected from 56 nurses using pre designed, pre tested and self developed Performa. Data was analysed using descriptive statistics (frequency, percentage and mean) and inferential statistics (Spearman rho correlation test)

Results Regarding the overall knowledge, majority of the respondents $(76.78 \%)$ had good knowledge; $23.21 \%$ had average knowledge and none of them had poor knowledge on HIV/AIDS. Total median score was 27, 25 32 in knowledge and attitude respectively. There was no correlation between knowledge and attitude $(p>0.05)$

Conclusion It is essential for organising continuous in service education to get informed the nurses so as to create awareness especially on PMTCT concepts and refer, guide and intervene the women living with HIV/AIDS to avail the PMTCT services and influence the nurses to develop positive attitude the people living with HIV/AIDS

\section{P6.057 DECREASING THE PROPORTION OF PATIENTS LOST TO FOLLOW-UP AT AN OUT-PATIENT HIV CLINIC IN EASTERN UGANDA}


'M Kakinda, 'I C Opio, '2J K B Matovu. 'Children's AIDS Fund, Kampala, Uganda, ${ }^{2}$ Makerere University School of Public Health, Kampala, Uganda

Background We implemented a facility-based intervention aimed at reducing the proportion of patients lost to follow-up at an outpatient HIV clinic in Jinja district, Eastern Uganda, over a period of 6 months (February-July 2012)

Methods The intervention was implemented with the aim of decreasing the proportion of patients lost to follow-up from $1 \%$ $(23 / 2328)$ at baseline to $0.25 \%$ by July 2012 . Simple and innovative strategies were introduced into the HIV clinic. These included retraining of clinic staffs on the importance of patients keeping their appointments; development of new messages on keeping appointments; retrieving patient files a day before the day of appointment; sending two text message reminders a day to the clinic; and actively following up patients who had not kept their appointments through home-visiting. At each follow-up visit, reasons for the patients' failure to keep appointment were noted and information on age, gender, CD4 count (captured from patient records) and duration in HIV care was obtained. Data were fed into an IQ Care programme and analysed using Microsoft Excel.

Results The proportion of patients lost to follow-up decreased from $1 \%(23 / 2328)$ at baseline to $0.4 \%(9 / 2528)$ in 6 months. Men, those with a CD4 $>350$, those aged $<30$ years and those in care for $<1$ year had the biggest decline in proportion of patients lost to follow-up compared to other patients.

Conclusion The proportion of patients lost to follow-up declined by $60 \%$ through use of simple and innovative strategies introduced in the HIV clinic.

\section{P6.058 UNIVERSAL HIV SCREENING OF INMATES IN ISRAELI PRISONS: SHOULD THE POLICY BE UPDATED?}

doi:10.1136/sextrans-2013-051184.1211

'Z Mor, ${ }^{2} \mathrm{H}$ Vider, ${ }^{3,4} \mid \mathrm{G}$ Grotto, ${ }^{5} \mathrm{D}$ Tischler Aurkin. ${ }^{1}$ Ministry of Health, Ramla, Israel; ${ }^{2}$ ssraeli prison Services, Ramla, Israel; ${ }^{3}$ Ministry of Health, Jerusalem, Israel; ${ }^{4} B e n$ Gurion University, Beer Sheva, Israel; ${ }^{5}$ sraeli Prison Services, Ramla, Israel

Background HIV rates among inmates are mostly higher than the general population. Israel is a relative low-HIV prevalence country (107:100,000 population, 2011 data). All criminal inmates incarcerated in prisons/gaols at the Israeli Prison Services (IPS) are routinely screened for HIV. This retrospective study evaluates the necessity of the current policy of universal HIV-testing policy.

Methods We reviewed all inmates diagnosed with HIV/AIDS upon incarceration in Israel between 2003 and 2010, and assessed their risk-behaviours and the date of diagnosis.

Results During the 8 years follow-up, 108,866 new criminal inmates were incarcerated in 31 correctional facilities, and it is estimated that $95 \%$ of those were tested for HIV. Of those, $201(0.2 \%)$ were tested positively with HIV, in a direct testing cost of 622,000 (US\$).

Of all $201 \mathrm{HIV}$-infected inmates, 118 (58.7\%) were intra-venous drug-users (IVDU), 55 (27.4\%) originated in high-prevalence countries, $13(6.5 \%)$ were men who have sex with men (MSM), 12 (6.0\%) were heterosexuals not originating in endemic country, $2(0.1 \%)$ the risk-group was undetermined and one $(0.5 \%)$ was infected vertically.

Of all 201 HIV-infected inmates, 157 (78.2\%) were diagnosed in the community, prior to their imprisonment, and were re-tested in prison; while $44(21.8 \%)$ were firstly diagnosed in prison. Of those 44 inmates, $25(56.8 \%)$ were IVDU, $13(29.5 \%)$ originated in endemic country, three $(9.1 \%)$ were MSM and in two $(4.5 \%)$ the risk-group was not determined.

Conclusion HIV-infection rate is prison is twice higher than the general population. The majority $(98.5 \%)$ of all inmates was diagnosed prior to their incarceration or had a key risk-behaviour expos- ing them to HIV. Therefore, questioning each new inmate upon incarceration about previous HIV-diagnosis, and targeted testing for other inmates who are IVDU, MSM or originating in endemic countries for HIV can detect almost all HIV-infected prisoners, presuming they respond reliably.

\section{P6.059 VERTICAL TRANSMISSION OF HIV LESS THAN HALF AMONG MOTHERS BELONGING TO MOTHER-SUPPORT GROUPS (MSG) COMPARED TO NON-MEMBER MOTHERS AT HEALTH CENTRES IN TIGRAY, ETHIOPIA}

doi:10.1136/sextrans-2013-051184.1212

'M Abraha, 'T A Kahsu, ${ }^{2} \mathrm{H}$ Godefay, 'E Konings. 'Management Sciences for Health, Addis Ababa, Ethiopia, ${ }^{2}$ Tigray Regional Health Bureau, Mekele, Ethiopia

Background Mother-support groups (MSGs) are used at hospitals and health centres in Ethiopia to increase uptake of and adherence to PMTCT and reduce vertical transmission of HIV. A study at Dessie Referral Hospital showed that mothers belonging to MSGs had lower vertical transmission rates. The impact on mothers seen at health centres is not known.

Methods Data on the outcome of MSGs on vertical transmission were collected from 15 health centres in Tigray between 2008 and 2011. Data sources included MSG registration log books, regional laboratory DNA/PCR results, and the ART enrollment register. We compared HIV status among babies born to mothers belonging to MSG and those whose mothers did not participate in MSG.

Results A total of 848 HIV-exposed infants (HEIs) were registered between 2008 and 2011. Among 240 HEIs born to mothers enrolled in MSG, $87.5 \%$ were tested for HIV and $5.2 \%$ were HIV-positive. Among the 608 HEIs whose mothers were not enrolled in MSGs, $44.7 \%$ were tested and $11.4 \%$ tested HIV-positive (OR $=0.43$ ).

Conclusions The odds of testing positive were $57 \%$ lower among HEIs whose mother was enrolled in an MSG compared to those whose mother was not enrolled. The better outcome appears to be due to the support their mothers received from MSGs resulting in better PMTCT and adherence practises as well as high rates of institutional delivery. We are currently collecting more data on PMTCT regimen, HEI testing by age and place of delivery to verify this hypothesis.

\section{P6.060 PATIENT OR PROVIDER REFERRAL FOR CHLAMYDIA - WHAT IS THE COST AND IS IT WORTH IT? A COST COMPARISON OF ALTERNATIVE STRATEGIES}

doi:10.1136/sextrans-2013-051184.1213

${ }^{1}$ T Roberts, ${ }^{2 \mathrm{H}}$ Mistry, 'M Rossello-Roig, ${ }^{3} \mathrm{G}$ Rait, ${ }^{4} \mathrm{~J}$ Dodds, ${ }^{5} \mathrm{~S}$ Lanza, ${ }^{4} \mathrm{C} S$ Estcourt, ${ }^{6} \mathrm{M}$ Symonds, ${ }^{5} \mathrm{~J}$ A Cassell. 'University of Birmingham, Birmingham, UK; ${ }^{2}$ University of Warwick, Coventry, UK; ${ }^{3}$ University College London, London, UK; ${ }^{4}$ Queen Mary, University of London, London, UK; ${ }^{5}$ Brighton and Sussex Medical School, Brighton, UK; ${ }^{6}$ Barts Health NHS Trust, London, UK

Background Partner notification (PN) is an essential element of STI control. Typically partner notification has been supported by specialist health advisors based in GUM clinics, but recently the role has been extended to community based Chlamydia screening officers (including primary care). We aimed to explore and compare costs of various approaches to $\mathrm{PN}$ for Chlamydia in different settings.

Methods We compared costs of being offered one of five approaches to partner notification from the health service perspective:

1. Routine specialist clinic PN (patient referral including infection specific information, and advice that the sex partner should attend clinic for testing and treatment)

2. Accelerated Partner Therapy (APT Hotline): nurse initiated $\mathrm{PN}$ at the general practise followed by telephone assessment of sex partner by clinic-based nurse qualified health adviser; 